\title{
Tres Años de Vigilancia de la Sensibilidad in vitro de Hemophilus influenzae Causantes de Infecciones Pediátricas
}

\author{
T.M. Lucia Reyes M.; Dra. Valeria Prado J.'
}

\author{
Three Years Surveillance of the Susceptibility of \\ Haemophilus Influenzae Isolated From Pediatric Infections
}

\begin{abstract}
The increasing clinical importance of Haemophilus influenzae ( $\mathrm{H}-\mathrm{i}$ ) in severe infections and the emergence of resistant strains to the most commonly used antibiotics lead us to set up an in vitro susceptibility surveillance along the years 1983 through 1985. Ninetytwo $\mathrm{H}-\mathrm{i}$ strains isolated from patients of four hospitals at urban Santiago, Chile, were included ( $n=26$ in $1983 ; n=27$ in $1984 ; n=39$ in 1985). The isolates came from cerebrospinal fluid specimens $(n=15)$, blood cultures $(n=12)$, middle ear aspirates $(n=8)$, eye secresions $(n=14)$, pleural or traqueal filuids $(n=14)$, and other places $(n=3)$. The in vitro activity of Ampicillin, Chloramphenicol, Cefotaxime and Cefriaxone (MIC 90 in $\mu / \mathrm{g} / \mathrm{ml}$ ) were, in each case $50,6.25,0.19$ and $0.78 \mathrm{jg} / \mathrm{ml}$ respectively. Resistance to Ampicilin fluctuated from $23.2 \%$ in the first year to $11,1 \%$ in the second and to $30.8 \%$ in the third year of this study, and was allways mediated by a $\beta$ Lactamase. Eight $\mathrm{H}-\mathrm{i}$ strains $(8.7 \%$ were resistant to Chloramphenicol, and in $7 / 8(7.6 \%$ this fact was asociated with resistance to Ampicillin (including bacteriae isolated from cerebrospinal fluid, pleural and middle ear aspirates). Ceftriax one and Cefotaxime were active against all the strains of $H-i$ this studied.

(Key words: Haemophilus influenzae, Antibiotics. Sensibility. Ampicillin. Chloramphenicol. Ceftrinxone. Cefolaxime).
\end{abstract}

La importancia clínica creciente de Hemophilus influenzae (Hi) en pediatría como causante de infecciones severas nos ha motivado para mantener una vigilancia del perfil de sensibilidad de cepas de Hi aisladas de pacientes con diferen. tes afecciones durante los años 1983-84 y 85, a los antimicrobianos de primera elección como Ampicilina y Cloramfenicol, asi como también a otros de alternativa como cefalosporinas de tercera generación $1,2,3,4,5$.

Estudios realizados en el Area Oriente de Santiago han demostrado que Hi es el responsable del $33 \%$ de los casos de meningitis purulenta en niños, (Banfi A. y col. Resúmenes II Congreso Infectologia) y aproximadamente del $44 \%$ de las otitis medias agudas en lactantes ${ }^{4}$.

En los últimos años, a partir de 1974, se ha detectado en forma progresiva y constante la emergencia de cepas de $\mathbf{H i}$ resistentes a Ampicilina en diferentes países del mundo6, 7, 8.9, 10 con una prevalencia entre $5 \%$ y $13 \%$. Esta resis-

1. Unjdad de Microbiologia. División Ciencias Médicas Oriente, Facultad de Medicina, Universidad de Chile. tencia está dada principalmente por la producción de una enzima betalactamasa del tipo TEM, la cual es codificada por un plasmidio que es transferible por conjugación ${ }^{7}$; este plasmidio tiene características idénticas a otros observados en enterobacterias por lo cual se ha planteado la posibilidad de intercambios genéticos in vivo entre Hi y enterobacterias.

Por otra parte también se han encontiado cepas de $\mathrm{Hi}$ resistentes a Ampicilina, que no producen la enzina betalactamasa, en estas cepas se ha evidenciado una modificación de las proteínas $3 \mathrm{a}$ y $3 \mathrm{~b}$ de la membrana externa de $\mathrm{Hj}$ que impide la fijación de antibióticos betalactámicos ${ }^{11}$, haciéndose el microorganismo me diante este mecanismo, impermeable a la Ampicilina.

En la actualidad, Cloramfenicol es la alternativa terapéutica en niños con infecciones sistėmicas por $\mathrm{Hi}$, pero desafortunadamente desde 1978, han aparecido cepas de $\mathrm{Hi}$ resistentes a este antimicrobiano; esta resistencia está dada fundamentalmente por la presencia de una enzina acetil transferasa, codificada también por un plasmidio ${ }^{12}$. Existen numerosas publicaciones que demuestran que ella va unida con resistencia 
de Ampicilina y Tetraciclina mediante el acoplamiento de genes de resistencia e integración de ellos en un plasmidio único, transferible, con peso molecular de $34 \times 10^{6}$ daltons, ${ }^{13}, 14,15$ \}, La capacidad de transferencias del plasmidio le otorga, al fenómeno, posibilidades de amplificación.

Tambien se han descrito cepas de $\mathrm{Hi}$ resistentes a Cloramfenicol, no productoras de la enzima acetil transferasa. Esta resistencia obedecería a una alteración de la permeabilidad de la membrana externa, por disminución de una proteína de 40 kilo-daltons ${ }^{16}$.

Nuestro interés es mostrar las variaciones y características de la sensibilidad in vitro de cepas de Hemophilus influenzae aislados en nuestro medio en los últimos 3 años, como un antecedente que debe considerar el pediatra en el manejo terapéutico de infecciones graves por $\mathrm{Hi}$.

\section{MATERIAL Y METODO}

Se estudió un total de 92 cepas de Haemophilus aisladas durante los últimos 3 años de procesos infecciosos en pacientes pediátricos hospitalizados en diferentes centros asistenciales: Hospital Luis Calvo Mackenna $(n=48)$; Hospital Roberto del Río $(n=27)$; Hospital San Juan de Dios ( $\mathbf{n}=7$ ); Hospital Exequiel González Cortés $(n=9)$; Conin San Felipe $(n=1)$. Se incluyeron 26 cepas aisladas de 1983,27 durante 1984 y 39 cepas durante 1985.

Las fuentes de aislamiento de estas cepas fueron: 50 de liquido cefalorraquídeo (LCR), 12 de hemocultivos, 8 de aspirados de oido medio, 14 de secreciones oculares, 3 de aspirados tra. queales, 2 de líquido pleural y 3 cepas de quemadura, celulitis y pus de injerto respectivamerte (Tabla 1). La identificacion de las cepas de Hi se efectuó de acuerdo con criterios internacionales aceptados 17 .

Se determinó la producción de betalactamasa en cada cepa mediante la técnica de Nitrocefina (cefalosporina cromógena $)^{17}$.

Se determinó la concentración inhibitoria minima (CIM) de los siguientes antimicrobianos: Ampicilina, Cloramfenicol, Ceftriaxona y Cefotaxima, siguiendo la técnica descrita en una publicación anterior ${ }^{9}$.

Para comparar los resultados de la actividad de los antimicrobianos se utilizaron los siguientes parámetros: CIM 50 (CIM en mcg/ml que es capaz de inhibir el desarrollo del $50 \%$ de las cepas) y CIM 90 (CLM en $\mathrm{mcg} / \mathrm{ml}$ que es capaz de inhibir el desarrollo del $90 \%$ de las cepas). Se definió el porcentaje de cepas sen. sibles considerando como valor de corte 3 mcg/ml para Ampicilina, 8 para Cloramfenicol y 16 para Ceftriaxona y Cefotaxima.

\section{RESULTADOS}

En la Tabla 1 se observa la distribución de las 92 cepas de Hemophilus de acuerdo con la edad de los pacientes $y$ el origen del aislamiento.

Podemos ver que $68,5 \% 63$ de las cepas corresponden a nifios menores de 12 meses y que hay un porcentaje importante de cepas aisladas de niños con meningitis hasta los 2 años de edad. El $54,3 \%$ ( 50 de las cepas estudiadas corresponden a casos de meningitis purulenta y $13 \% 12$ fueron aisladas de hemocultivos.

En la Tabla 2 se muestra la correlación de la producción de betalactamasa con el lugar de aislaniento de Haemóphilus. Se encontraron cepas productoras de esta enzima en todos los sitios de aislamiento, con excepción de 14 cepas de

Tabla 1

Material de Estudio. 92 Cepas Hemophilus Influenzae

1983-84-85

ORIGEN AISLAMIENTO DE HAEMOPHILUS

\begin{tabular}{|c|c|c|c|c|c|c|c|c|}
\hline EDAD & $\underset{\text { cepas }}{\mathrm{N}^{\circ}}$ & LCR & $\begin{array}{l}\text { Hemocul- } \\
\text { tivo }\end{array}$ & Oido & Ocular & Traqueal & Pleural & $\begin{array}{l}\text { Celulitis } \\
\text { Pus } \\
\text { injerto }\end{array}$ \\
\hline$<6$ meses & 22 & 11 & 3 & 3 & $s$ & - & - & - \\
\hline $6-12$ meses & 41 & 23 & 5 & 4 & 5 & 2 & - & 2 \\
\hline 13-24 meses & 18 & 10 & 3 & 1 & 3 & - & 1 & - \\
\hline 2-7 años & 11 & 6 & 1 & - & 1 & 1 & 1 & 1 \\
\hline TOTAL & 92 & 50 & 12 & 8 & 14 & 3 & 2 & 3 \\
\hline
\end{tabular}


secreciones oculares y 3 cepas procedentes de casos de celulitis e injerto respectivamente. De 50 cepas aisladas de LCR $24 \%$ producían la enzima betalactamasa, como ocurría también con $25 \%$ de las 12 cepas aisladas de hemocultivos. Durante estos 3 años de vigilancia, la prevalencia de $\mathrm{Hi}$ productora de betalactamasa y por ende resistentes a Ampicilina fluctuó entre 11,1\% y 30,8\%, con un promedio de $22,9 \%$.

En la Tabla 3 se compara la actividad "in vitro" de Ampicilina, Cloramfenicol, Ceftriaxona y Cefotaxima sobre las 92 cepas de Haemóphilus.

En el caso de Ampicilina, 76\% de las cepas (70/92) fueron sensibles con CIM 50 de 0,78 $\mu \mathrm{g} / \mathrm{ml}$ y CIM 90 de $50 \mu \mathrm{g} / \mathrm{ml}$. Entre las cepas resistentes se detectó producción de betalactamasa en 21 de 22.

Cloramfenicol resultó ser activo en $91,3 \%$ de las cepas con CIM 50 de $0.39 \mu \mathrm{g} / \mathrm{ml}$ y CIM 90 de $6,25 \mu \mathrm{g} / \mathrm{ml}$.

Las cefalosporinas de tercera generación (Ceftriaxona y Cefotaxima) mostraron actividades 20 y 10 veces mayores que Ampicilina y Cloramfenicol respectivamente, reflejadas en CIM $50 \leqslant 0,04$ y CIM 90 de 0,19 y $0,78 \mu \mathrm{k} / \mathrm{ml}$, resultando todas las cepas sensibles a ellas.

En la Tabla 4 se analiza el patrón de resistencia de 21 cepas de Haemóphilus, el 14,1\% (13 cepas) mostraron resistencia única a Ampicilina y $1,1 \%$ a Cloramfenicol.

Tabla 2

Prevalencia de Cepas de Haemophilus Influenzae

Productoras de Betalactamasa

\begin{tabular}{|c|c|c|c|c|c|c|c|c|}
\hline \multirow[b]{2}{*}{ ORIGEN } & \multicolumn{2}{|c|}{1983} & \multicolumn{2}{|c|}{1984} & \multicolumn{2}{|c|}{1985} & \multicolumn{2}{|c|}{ TOTAL } \\
\hline & No cepas ${ }^{T}$ & $|\%|$ & $N^{0}$ cepas 1 & $(\%)$ & $\mathrm{N}^{0} \propto \mathrm{pas}^{1}$ & $(\%)$ & $\mathrm{N}^{0}$ cepas 1 & $(\%)$ \\
\hline LCR & $2 / 11$ & & $1 / 15$ & & $9 / 24$ & . & $15 / 50$ & (24) \\
\hline Hemocultivo & - & & $1 / 4$ & & $2 / 8$ & & $3 / 12$ & (25) \\
\hline S. ótica & $2 / 7$ & & $1 / 1$ & & - & & $3 / 8$ & (37.5) \\
\hline S. ocular & $0 / 5$ & & $0 / 4$ & & $0 / 5$ & & $0 / 14$ & \\
\hline S. traqueal & $0 / 1$ & & - & & $1 / 2$ & & $1 / 3$ & (33.3) \\
\hline S. pleural & $2 / 2$ & & _ & & - & & $2 / 2$ & $(100)$ \\
\hline Otras* & - & & $0 / 3$ & & - & & $0 / 3$ & 0 \\
\hline & $6 / 26$ & $(23,2$ & $3 / 27$ & $(11,1$ & $12 / 39$ & $(30,8$ & $21 / 92$ & $(22,9)$ \\
\hline
\end{tabular}

* Injer to, celulitis, quemadura

1 No cepas betalactamasa +/total cepas estudiadas.

Tabla 3.

Sensibilidad In Vitro CM $(\nu \mathrm{g} / \mathrm{ml})$ de 92 Cepas de Haenrophilus Frente

a 4 Antimicrobianos

\begin{tabular}{lcccc}
\hline Antimicrobiano & $\begin{array}{c}\text { CIM } 50 \\
\mu g / m l\end{array}$ & $\begin{array}{c}\text { CIM } 90 \\
\mu g / m l\end{array}$ & $\begin{array}{c}\text { Cepas } \\
\text { No }\end{array}$ & $\begin{array}{c}\text { Sensibles* } \\
\%\end{array}$ \\
\hline Ampicilina & 0,78 & 50 & $70 / 92$ & 76 \\
Cloramfenico & 0,39 & 6,25 & $84 / 92$ & 91,3 \\
Ceftriaxona & $\leqslant 0,04$ & 0,19 & $92 / 92$ & 100 \\
Cefotaxima & $\leqslant 0,04$ & 0,78 & $85 / 85$ & 100 \\
\hline
\end{tabular}

* $\mathrm{CIM}$

$\begin{array}{ll}\text { Ampicilina } & <3 \mu \mathrm{g} / \mathrm{ml} \\ \text { Cloramfenicol } & <8 \mu \mathrm{g} / \mathrm{ml} \\ \text { Ceftriaxoma } & <16 \mu \mathrm{g} / \mathrm{ml} \\ \text { Cefotaxima } & <16 \mu \mathrm{g} / \mathrm{ml}\end{array}$


Patrón de Resistencia de 21 Cepas de Haemophilus

\begin{tabular}{lccccccc}
\hline Resistente a: & Total & LCR & $\begin{array}{c}\text { Hemocul- } \\
\text { tivo }\end{array}$ & S.pleural & S. oído & S. traqueal & S. ocular \\
\hline Ampicilina & 13 & 8 & 3 & - & 1 & 1 & - \\
$\begin{array}{l}\text { Cloramfericol } \\
\text { Ampicilina } y\end{array}$ & 1 & - & - & - & - & - & 1 \\
Cloramfenicol & 7 & 4 & - & 2 & 1 & - & - \\
\hline
\end{tabular}

Podemos observar que 7 de las cepas estudiadas $(7,6 \%)$ mostraron resistencia combinada a Ampicilina y Cloramfenicol (4 de ellas fueron aisladas de casos de meningitis) $3 / 26$ cepas aisladas en $1983,1 / 27$ en 1984 y $3 / 37$ en 1985 .

\section{COMENTARIOS}

Hemóphilus influenzae (Hi) es causa frecuente de enfermedades infecciosas en la edad infantil; algunas observaciones indican que $95 \%$ de los niños menores de 5 años sufren por lo menos una infección por este agente ${ }^{18}, 19$

Las 92 cepas incluidas en este estudio durante el período 1983-85 fueron obtenidas de proce. sos patológicos en pacientes pediátricos del área Oriente, Norte Occidente y Sur de Santiago por lo cual estimamos que la información obtenida refleja lo que está sucediendo en Santiago respecto a sensibilidad de $\mathrm{Hi}$, aunque no podría ser extrapolado al resto del país.

La mayor proporción de cepas de $\mathrm{Hi}$ de este estudio fue aislada de niños menores de 12 meses, periodo en el cual dentro de la edad pediátrica son más frecuentes las infecciones graves por $\mathrm{Hi}$.

El estudio de la sensibilidad a los antimicrobianos inclujdos indica que $76 \%$ del total de cepas son sensibles a Ampicilina y el porcentaje de resistencia de estos 3 años fluctuó entre 11 y $30 \%$. De las cepas resistentes todas, con excepción de una, fueron productoras de betalactamasa, por lo tanto sigue siendo útil e importante la detección de esta enzima como un método rápido de detectar resistencia a Ampicilina.

La resistencia a Cloramfenicol es de baja frecuencia, un $91,3 \%$ de las cepas fueron sensibles a Cloramfenicol, antimicrobiano que sin embargo, no se puede considerar como alternativa segura de la Ampicilina ya que 4 cepas de LCR, 2 aișladas de empiema pleural y una de oído medio fueron resistentes a ambas drogas, fenómeno que tambjén ha sido descrito en centros extranjeros $13,14,15$ por lo cual se hace necesario contar con una técnica rápida para la detección de la enzima Cloramfenicol acetil transferasa. Esta técnica ya ha sido descrita ${ }^{20}$ y se ha establecido de rutina en algunos centros, de modo que frente a infecciones sistémicas graves por Hi como meningitis, se determina la producción de betalactamasa y de acetil transferasa to cual le permite dirigir en forma más racional la terapia.

Las cefalosporinas de tercera generación (Ceftriaxona y Cefotaxima) mantienen una excelente actividad in vitro frente a $\mathrm{Hi} 20$ a 10 veces superior a Ampicilina y Cloramfenicol y todas las cepas fueron sensibles a ellas, como ya habíamos detectado previamente 9 . Ensayos clínicos utilizando Ceftriaxona en el tratamiento de meningitis purulenta por $\mathrm{Hi}$ y otros agentes, realizados en nuestro medio (Prado V. y col. Resímenes Il Congreso Panamericano de Infectologia, Buenos Aires 1984, pág. 78) (Prado V. y col. Resúmenes II Congreso Chileno de Infectología, pág. 52) han mostrado una buena respuesta clínica y bacteriológica, con resultados semejantes a los obtenidos con la terapia habitual, lo cual ha sido observado también por investigadores extranjeros $20,21,22$, 23

Igualmente los estudios clínicos en los cuales se ha utilizado cefotaxima en meningitis han demostrado su utilidad $24,25,26,27$.

El perfil actual de la sensibilidad de $\mathrm{Hi}$ en el área metropolitana mantiene la vigencia de Am. picilina y/o Cloramfenicol como terapis de primera elección en el tratamiento de las infecciones por este agente, pero hace aconsejable un estudio bacteriológico activo y dinámico incluyendo đeterminación de sensibilidad, de cada cepa de $\mathbf{H j}$ que está provocando infecciones sistémicas graves, Frente a cepas de Hi con resistencia combinada tanto a Ampicilina como a Cloramfenicol las cefalosporinas de tercera generación cons. tituyen una buena altemativa. 


\section{RESUMEN}

La importancia clínica creciente de Hemophilus influenzae ( $\mathrm{Hi}$ ) en infecciones severas $\mathrm{y}$ la aparición de cepas resistentes a los antimicrobianos de elección motivó una vigilancia de la sensibilidad in vitro durante los años 1983, 1984 y 1985. Se incluyeron 92 cepas de $\mathrm{Hi}$, aisladas en 4 hospitales de Santiago ( 26 en 1983, 27 en 1984 y 39 en 1985), que provenian de líquido cefalorraquídeo $(\mathrm{n}=50)$, hemocultivos $(\mathrm{n}=12)$, oido medio $(n=8)$, secreciones oculares $(n=$ 14), secreciones traqueales o pleurales $(n=5)$ y de otras secreciones $(n=3)$. La actividad in vitro (ClM 90 en $\mu \mathrm{g} / \mathrm{ml}$ ) de Ampicilina, Cloramfenicol, Cefotaxima y Ceftriaxona fue $50 ; 6,25 ; 0,19$ y $0,78 \mu \mathrm{g} / \mathrm{ml}$ respectivamente. La resistencia a Ampicilina fluctuó entre $23,2 \%, 11,1 \%$ y $30,8 \%$ en los 3 años, con un promedio de $24 \%$, y fue mediada por betalactamasa en todas las cepas con excepción de una; ocho cepas $(8,7 \%)$ resultaron resistentes a Cloramfenicol, en $7 / 8(7,6 \%)$ simultáneamente con Ampicilina (cepas de LCR, hiquido pleural y oído medio). Cefotaxima y Ceftriaxona resultaron activas frente al $100 \%$ de las cepas.

\section{AGRADECIMIENTOS}

Agradecemos a los doctores Walter Ledermann, In* geborg Prenzel, María Eugenta Pinto, Jorge Vergara y María Teresa Siri, el suministro de cepas de Hemophilus.

\section{REFERENCIAS}

1. Herson V., Todd $J .:$ Prediction of morbidity in Hemophilus influenzae meningítis. Pediatrics 59: 35. 1977.

2. Dofari A., A mar B, y col.: Systemic Haemophilus influenzae disease. An over view. J. Pediatr, 94: 355,1979 .

3. Rubin L, Moxon R.: Pathogenesis of bloodstream invasion with Haemophilus influenzae tipe b. Infect. Inmun. 41 : $280,1983$.

4. Sìri M.T., Banfi A. y col: Variación de s etiología de otitis media aguda en lactantes durante un período de 4 años de estudio (1979-1983). Rev. Chit. Infectología 2: 77, 1984.

5. Ledermann W., Cohen J., Siri M.T.: Incidencia de Haemophilus influenzae productoras de betalactamasa en infecciones pediátricas Rev. Chil. Pediatr. 54: $166,1983$.

6. Schiffer M.S., Schneerson R. y col: Clitucal, bacteriological and immunological characterisation of ampecillin-resistant Haemophilus influenzae tipo b. Lancet. August 3: 257. 1974.
7. Madeiros A, O'Brien T.: Ampicilin-resistant Haemophilus influenzae type b possesing a TEM type beta laetamase but little permeability barrier to ampicilin. Lancet 29: 716, 1975.

8. Kahn W. Roso S. y col. Haemophilus influenzae type b resistant to ampicillin. JAMA 229: 298, 1974.

9. Reyes L., Prado V., Siri M.T.: Perfil de sensibilidad a los antimicrobianos de Haemophilus influenzae aislados de patología infantil. Rev. Chil, Pediatr. 55: $\$ 4,1984$.

10. Lerman S.J., Brunken J.M., Bollinger H.: Prevalence of Ampicillin resistant strains of Haemophilus influenzae causing systemic infection. Antimicrob. Agents Chenother 18:474, 1980.

11. Parr T., Bryon L.: Mechanjsm of resistance of an ampicillin-resistant, B lactamase-negative clinical isolate of Haemophilus influenzae type $b$ to betalactamantibiotics. Antimicrob. Agents Chemother 25: $747,1984$.

12. Roberts M. Srueson C. $y$ col.: Characterization of chloramphenicol-resistant Haemophilus influenzae. Ant imicrob. Agents Chemother 18: 610, 1980.

13. Brvan L.E.: Transferable chloramphenicol and ampicillin resistance in a strains of Haemophilus influenzae. Antimicrob. Agents Chemother 14: 154, 1979.

14. Keniny J.F., Isburaj C.D. y col.: Meningitis due to Haemophilus influenzae type b resistant to both ampicillin and chloranphenicol. Pediatrics 66: 14, 1980.

15. Campos J., García Tornel S.: Susceptibility studies of multiply tesistant Haemophilus infuenzae isolated from pediatrics patiens and contacts. Antimicrob. Agents Chemothez 25: 706, 1984.

16. Burns J., Mendelman P. y col.: A permeability barrier as a mechanism of chloramphenicol re sistance in Haemophilus inffuenzae. Antimucrob. Agents Chemother 27: 46, 1985.

17. Lennette E.H.: Microbiología clínica, $3^{\mathrm{a}}$ Ed. Edjtorial Médica Panamericana, Buenos Aires 1982.

1B. Thomsberry C., Mc Dougal L.K.: Ampicillin resistant Haemophilus jnfluenzae 1. Incidence mechanism and detection. Postgrad Med. 71: 133, 1982.

19. Thortsberry C., Mc Dougal L.K.: Ampicilin resistant Hemophilus influenzae 2. Therapeutic considerations Post grad Med. 71: 1, 1982 .

20. Azemun P., Stull T., Roberts M., Smith A.: Rapid detection of chloramplienicol resistance in Haemophilus influenzae. Antimicrob Agents Chemother 20: 168, 1981.

21. Del Rio M.A., Chrane D., Shelton S. et al.: Ceftriaxone versus ampicillin and chiorampheniool for tratment of bacterial meningitis in children. Lancet 1: $1241,1983$.

22, Steele R.W., Bradsher R.W.: Comparison of Ceftrinxone with standard therapy for bacterial meningitis. J, Pediatr. 103: 138, 1983.

23. We Cracken G.M.: New concepts in the management of infants and children with meningitis. Ped. [nfect. Dis. 2: 51, 1983.

24. Wells T.G., Trang J.M., Brown A.L, et al.: Cefotaxime theraphy of bacterial meningitis in chijdren. J. Antimicrob. Chemother 14 (suppl B): 181, 1984.

25. Kafetzis D.A., Brater D.C. Kapiki A.M., Papas C.V. et al.: Treatment of severe neonatal infection with cefotaxime. Efficacy and pharmacokinetics. J. Pediats. 100: 483, 1982. 
26. Kobayashi Y., Morikawa Y., Honuta T. et al.: Clinical evaluation of cefotaxime in the treatment of purulent meningitis in children. Jap. J. Antibiot. 34: 946,1981 .
27. Borderon J.C., Prieur D., Huguet B.: Concentrations du cefotacime dam le liquide cephalorachidien chez des enfants atteints de meningite purulente. Now. Press Med. 10:580, 1981. 